\title{
Hunting for elusive multi-messenger transients with INTEGRAL
}

\author{
Volodymyr Savchenko*t \\ University of Geneva \\ E-mail: Volodymyr.Savchenko@unige.ch
}

\begin{abstract}
Recent years were marked by breakthrough observations of new multi-messenger and multiwavelength transients, often unveiling unanticipated and puzzling phenomena. Likely associated with most energetic processes in the dense region at the heart of peculiar supernovae, mergers of compact objects, or tidal disruption events, these events expose unique signatures in hard X-ray and gamma-ray emission.

I highlight recent pioneering observations of short energetic transients made with INTEGRAL, which is especially well-equipped to observe unpredictable, short-lived, and energetic hard X-ray and gamma-ray transients. It carries a collection of detectors that monitor the entire hard X-ray sky with over $80 \%$ duty cycle and is able to re-point to perform deep and sensitive hard X-ray observations of any large selected sky region. I discuss the observations of gamma-ray bursts, in particular in association with the gravitational wave events and high-energy neutrinos, and review how INTEGRAL observations of fast hard X-ray transients help to reveal mechanisms of the peculiar supernovae. Finally, I will discuss how the recent discoveries in the domain of multimessenger transients were made possible by a global effort to achieve a new degree of automation and interoperability.
\end{abstract}

The New Era of Multi-Messenger Astrophysics - Asterics2019

25 - 29 March, 2019

Groningen, The Netherlands

\footnotetext{
*Speaker.

${ }^{\dagger}$ on behalf of a larger collaboration: https://www.astro.unige.ch/cdci/integral-multimessenger-collaboration
} 


\section{Introduction}

Recent emergence of rapid optical and radio surveys accelerated the development of time domain astronomy. Furthermore, observations of gravitational waves and neutrinos enabled breakthrough multi-messenger observations of fundamental physical processes in space. Short and energetic events are generally easier to separate from the background than persistent sources, which is why the first truly multi-messenger signals were also detected as transient events.

The properties or even the nature of these new transient sources often remain uncertain, typically hinting towards particularily energetic transformations of compact objects: peculiar supernovae, mergers of compact objects, or tidal disruption events. An important perspective on these events is given by hard X-ray and gamma-ray emission: it tends to reveal a distinct emission component that points to the most dense and energetic regions at the heart of the source.

In this paper we highlight recent pioneering observations of short energetic transients made with INTEGRAL, which is especially well-equipped to observe unpredictable, short-lived, and energetic hard X-ray and gamma-ray transients. It carries a collection of detectors that monitor the entire hard X-ray sky with over $80 \%$ duty cycle and are able to re-point to perform deep and sensitive hard X-ray observations of a large selected sky region.

\section{INTEGRAL observatory}

INternational Gamma-Ray Astrophysics Laboratory [INTEGRAL 1] is an M-class ESA mission, operating since 2002. It is equipped to study hard X-ray and gamma-ray emission in the energy range from $3 \mathrm{keV}$ to more than $10 \mathrm{MeV}$. Photons in this energy range are typically produced in dense and energetic regions, through synchrotron radiation, compton scattering, nuclear decays, etc. Due to particularily low cross-section, this emission can reach out from the deepest regions of compact sources. On the other hand, photons with these energies are also particularily hard to intercept and characterize. INTEGRAL relies on a collection of heavy detectors, with large stopping power. To distinguish emission from different sources it employs a set of coding masks, projecting thoroughly designed patterns on the detectors. The coded masks are only projected within limited Fields of View (within $1000 \mathrm{deg}^{2}$ ), but due to exceptional penetrating power of photons above $100 \mathrm{keV}$, they reach the same detectors from any direction in the sky. Sometimes a nuissance this also implies that INTEGRAL has exceptional grasp of hard X-ray emission from any position in the sky. All-sky observations are especially important when the sources appear unexpectedly at random sky positions, preventing any scheduled observations with sensitive narrow-field instruments. Since multiple INTEGRAL instruments can be used in this fashion, it is possible to reconstruct sky direction by exploiting difference of sky sensitivity between different detectors. [2].

In addition, owing to its highly elongated orbit, INTEGRAL does not suffer from any occultation by the earch, unlike every one of the near-Earth spacecraft (like Fermi, AGILE, or Swift). INTEGRAL instruments are online up to $85 \%$ of the time, just to give a sufficient margin to avoid the damaging the instruments in the radiation belts. 


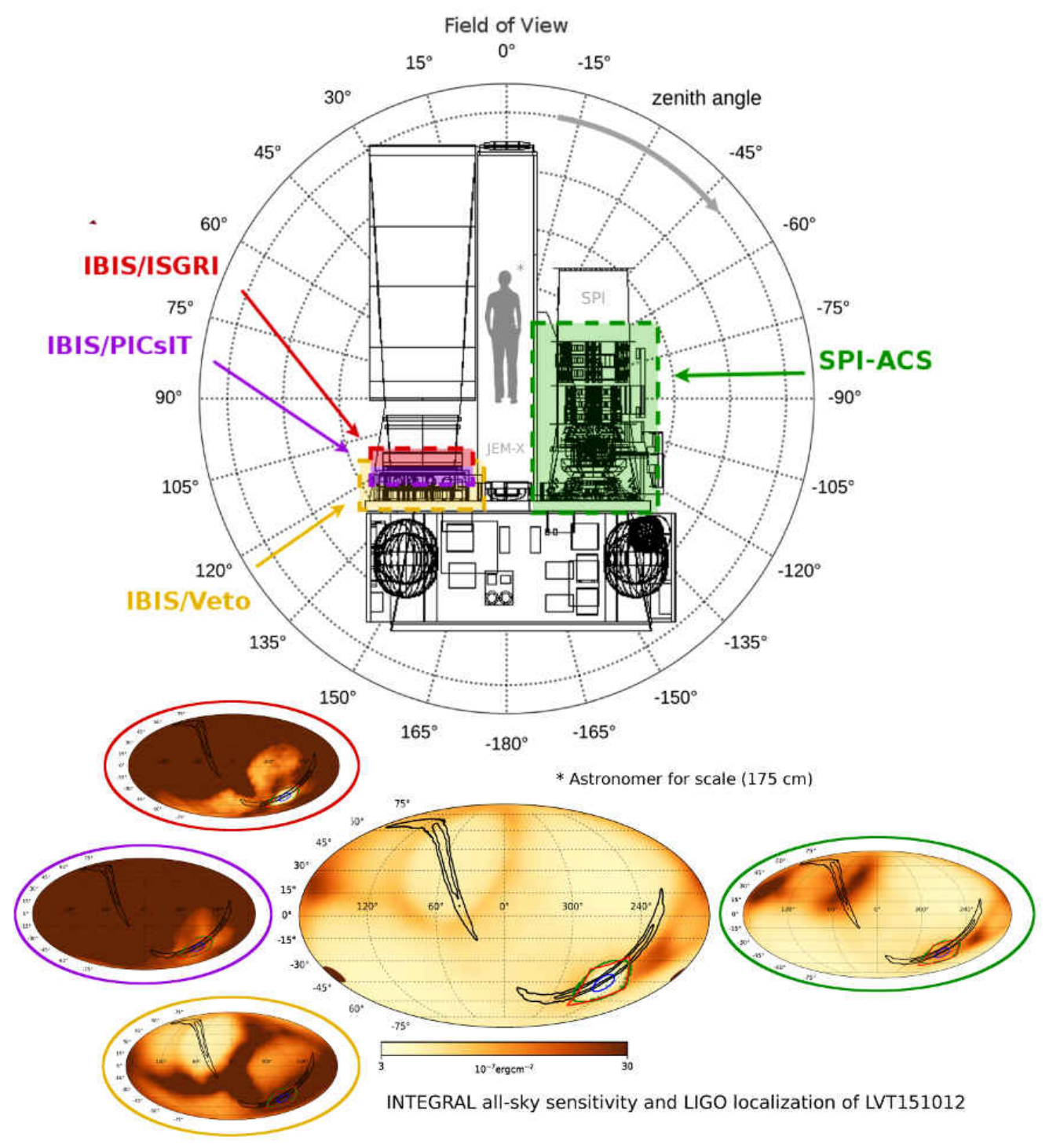

Figure 1: Schematic view of the INTEGRAL spacecraft, highlighting the all-sky instruments. A typical astronomer is shown for scale inside JEM-X monitor. The bottom panel shows contribution to the allsky sensitivity to any gamma-ray counterpart from GW151012 from different all-sky detectors on-board INTEGRAL (note the color coding matching the top panel), [see 2, for detailed study]

\section{Observations of GW transients}

Since 2015 LIGO detector network (later joined by also by Virgo) has been making groundbreaking observations of gravitation wave sources. The most clear GW signal events is emitted in short bursts just before coalescence of compact binary objects, such as black holes and neutron stars. It is not currently possible to pinpoint the distant compact binary before its collapse, and the localization provided by the GW network is still rather uncertain. This implies that the best observations close to the time of the events come from detectors which do not need to be pointed to a particular source, observing the whole sky at once. As described in the previous section, 
INTEGRAL, fits these requirements exceptionally well. From the very first GW detection, INTEGRAL has been performing unique and vital observations of the first GW transients - Binary Black Hole mergers. No gamma-ray signal was found by INTEGRAL from any of the BBH mergers $[2,3,4,5]$ (see Fig. 2). A report of a low-significance gamma-ray counterpart of GW150914 by Fermi/GBM[4, 6, 7, 8] sparked considerable interest in a possibility that such counterparts might be real, despite any prior expections. Implications of the INTEGRAL upper limits on the Fermi/GBM observations remain uncertain, owing to challenges in characterizing and comparing such a lowsignificance event between two instruments with only partially overlapping energy ranges.

The capacity of INTEGRAL to detect gamma-ray counterparts of the GW sources was highlighted by the detection of the first GRB associaed with a GW burst, GRB170817A Fig. 3. Owing to its superiour sensitivity and nearly uninterrupted observations, INTEGRAL was essentially guaranteed to observe it, while even Fermi/GBM, although being more sensitive that SPI-ACS to softer events, almost missed it due to the SAA passage (contributing to its $50 \%$ duty cycle).

First detection of GRB-GW source exceeded all but most optimistic expectations: it was thought that most of the short GRBs occur relatively large distances, well outside LIGO/Virgo reach. Instead, GRB170817A was a very nearby, very sub-luminous event. It did not quite fit in the basic GRB framework, and required extensions involving low-power wide-angle outflow, such as part of the stuctured jet, a cocoon inflated by provpagation a "normal" ultra-relativistic GRB jet $[9,10,11]$, or even delayed activity of a long-living remant of the merger - likely a young magnetar $[12,13,14]$.
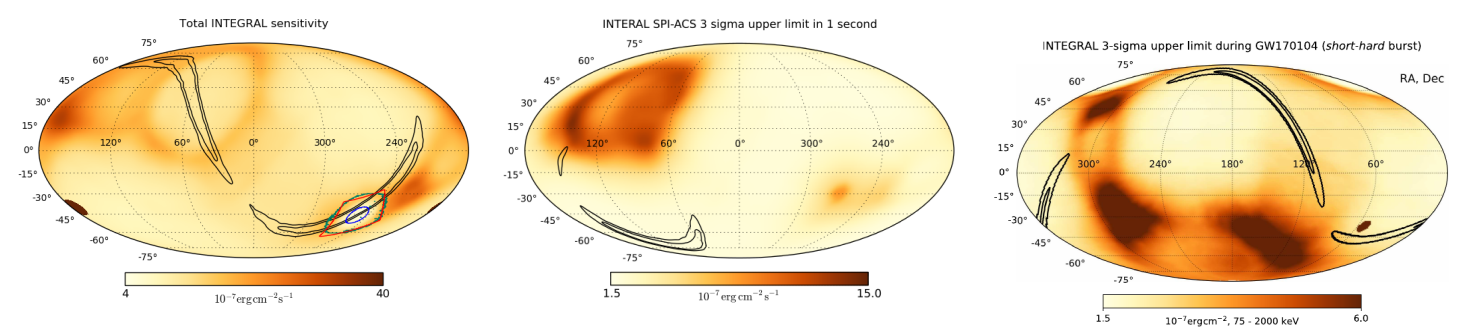

Figure 2: INTEGRAL upper limits on gamma-ray counterpart of several BBH mergers found by LIGO in realtime durting $\mathrm{O} 1$ and $\mathrm{O} 2[2,3,4,5]$.

\section{INTEGRAL detection of Hard X-ray component in AT2018cow}

Although the pivital role of INTEGRAL in observations of short impulsive gamma-ray transients has been firmly established, the searches of slowly decaying weak hard X-ray sources with its pointed coded mask instrument were always seen more in an explorative perspective. Until recently, no stellar-scale object has been detected from distances at which any GRB or GW sources are found. This has changed last year, when a coordinated real-time effort enabled the detection of AT2018cow, likely an exceptionally fast and luminous supernovae at $60 \mathrm{Mpc}$ [18, 19, 21, 20, 22].

\section{Conclusions}

Recent progress highlighted role of hard X-ray and soft gamma-ray emission in revealing inner workings of the relativistic sources of multi-messenger signals. More more fruitful observations 

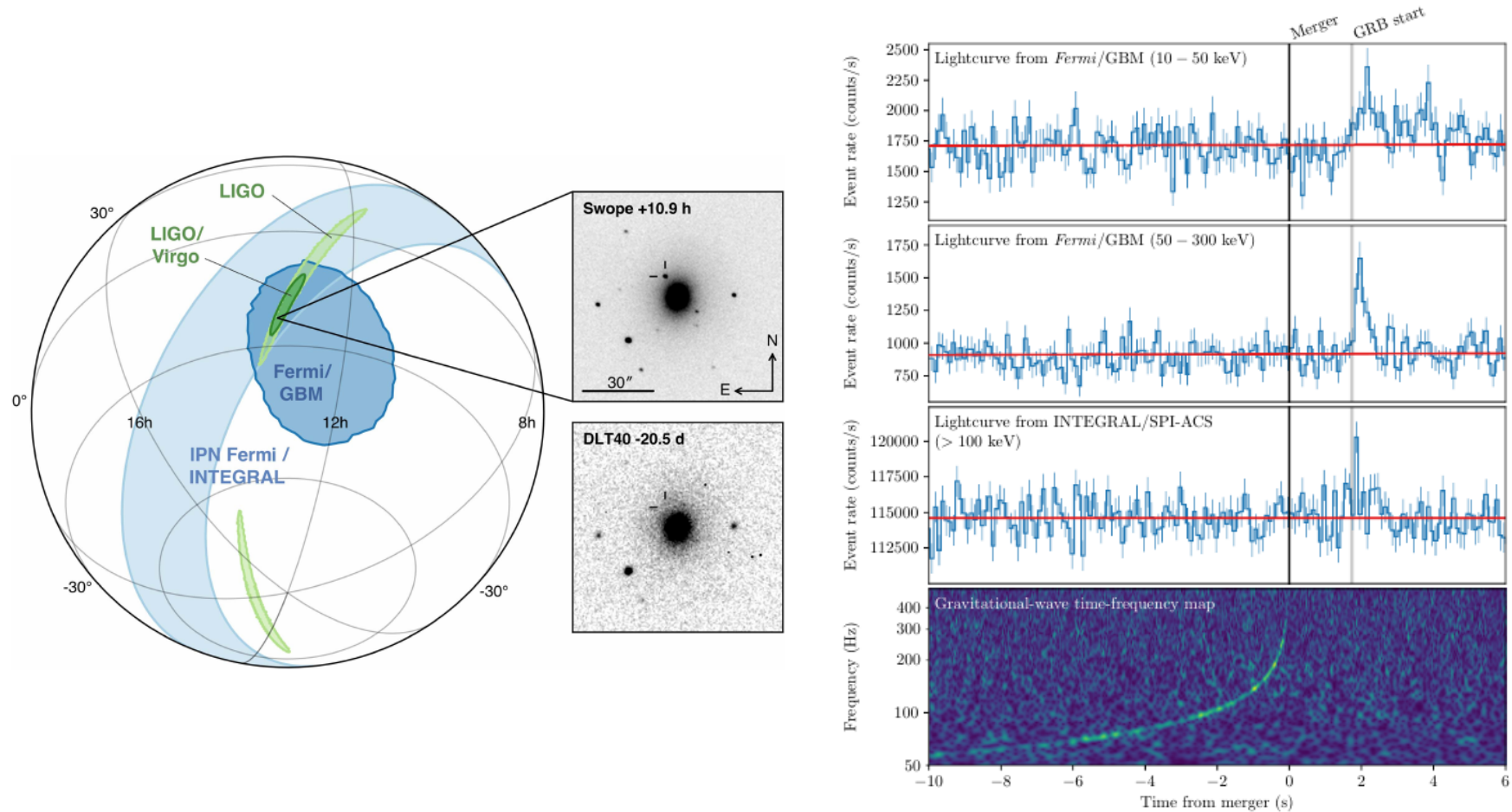

Figure 3: INTEGRAL and Fermi observation of GRB170817A, coincident with GW170817 [15, 16, 17]

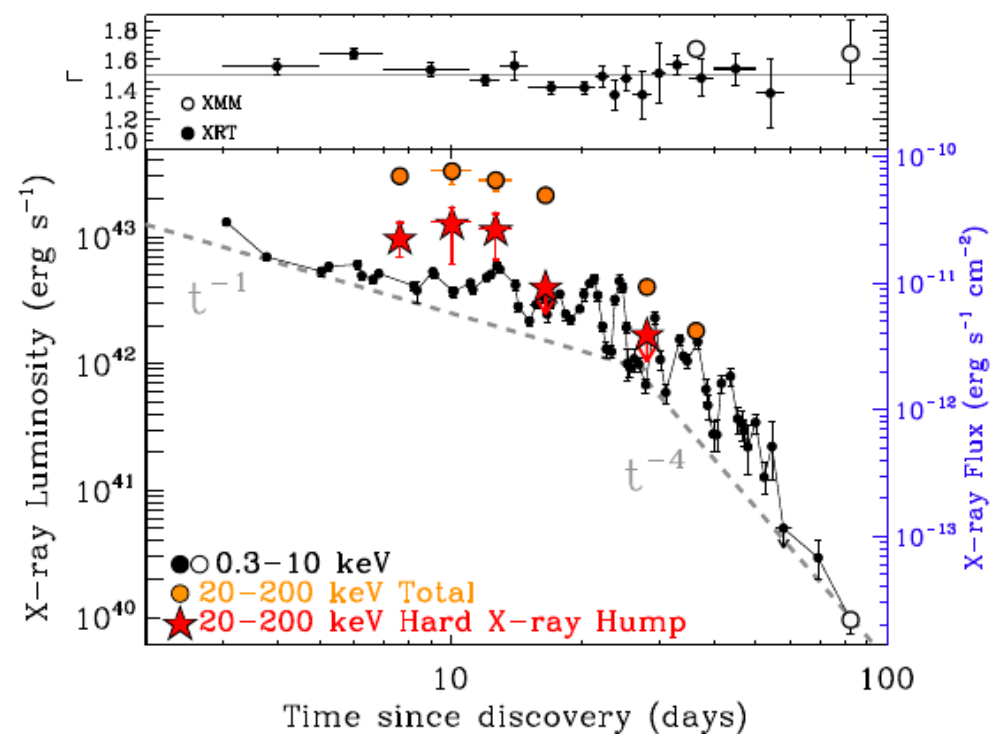

Figure 4: INTEGRAL, Swift, and NuSTAR observations of AT2018cow [18, 19, 20]. 
are being collected as multi-wavelength and multi-messenger observations are reaching new and puzzling kinds of sources. INTEGRAL features sufficient sensitivity to remain on the forefront of these synergystic studies for the years to come.

It is remarkable and important to note that recent discoveries in the domain of multi-messenger transients were made possible by a global effort to achieve a new degree of automation and interoperability, in which INTEGRAL is among the frontrunners, owing to the multiple synergystic efforts and developments maintained by Department of Astronomy at University of Geneva (in particular CDCI $[23,24])$ as well as ESAC ${ }^{1}$.

\section{References}

[1] C. Winkler, T. J.-L. T. J.-L. Courvoisier, G. Di Cocco, et al. The INTEGRAL mission. Astronomy \& Astrophysics, 411(1):L1-L6, 2003. ISSN 0004-6361. doi: 10.1051/00046361:20031288.

[2] V. Savchenko, A. Bazzano, E. Bozzo, et al. INTEGRAL IBIS, SPI, and JEM-X observations of LVT151012. Astronomy \& Astrophysics, Volume 603, id.A46, 15 pp., 603, 2017. ISSN 14320746. doi: 10.1051/0004-6361/201730572.

[3] V. Savchenko, C. Ferrigno, E. Bozzo, et al. INTEGRAL Observations of GW170104. The Astrophysical Journal, 846(2):L23, 2017. doi: 10.3847/2041-8213/aa87ae.

[4] J Greiner, J.M̃. Burgess, V Savchenko, and H.-F. Yu. On the Fermi-GBM Event $0.4 \mathrm{~s}$ after GW150914. 、apjl, 827:L38, 2016. doi: 10.3847/2041-8205/827/2/L38.

[5] V. Savchenko, C. Ferrigno, S. Mereghetti, et al. INTEGRAL upper limits on gamma-ray emission associated with the gravitational wave event GW150914. 2016. doi: 10.3847/20418205/820/2/L36.

[6] V. Connaughton, E. Burns, A. Goldstein, et al. Fermi GBM Observations of LIGO Gravitational Wave event GW150914. ArXiv e-prints, 2016.

[7] Maxim Lyutikov. Fermi GBM signal contemporaneous with GW150914 - an unlikely association. eprint arXiv:1602.07352, 2016.

[8] Shaolin Xiong. Is the GW150914-GBM really associated with the GW150914? 2016.

[9] Davide Lazzati, Rosalba Perna, Brian J. Morsony, et al. Late time afterglow observations reveal a collimated relativistic jet in the ejecta of the binary neutron star merger GW170817. Physical Review Letters, 120(24):241103, 2017. ISSN 0031-9007. doi: 10.1103/PhysRevLett.120.241103.

[10] N. Fraija, D. Lopez-Camara, A. C. Caligula do E. S. Pedreira, et al. Signatures from a Cocoon and an off-axis material ejected in a merger of compact objects: An analytical approach. eprint arXiv:1904.07732, 2019.

\footnotetext{
${ }^{1}$ https://www.cosmos.esa.int/web/esac-cmso/sepp
} 
[11] Ore Gottlieb, Ehud Nakar, Tsvi Piran, and Kenta Hotokezaka. A cocoon shock breakout as the origin of the \$ Igamma \$-ray emission in GW170817. Monthly Notices of the Royal Astronomical Society, 479(1):588, 2017. doi: 10.1093/mnras/sty1462.

[12] Kazumi Kashiyama, Kohta Murase, Imre Bartos, Kenta Kiuchi, and Raffaella Margutti. Multi-Messenger Tests for Fast-Spinning Newborn Pulsars Embedded in Stripped-Envelope Supernovae. The Astrophysical Journal, 818(1):94, 2015. doi: 10.3847/0004-637X/818/1/94.

[13] W. H. Gao and Y. Z. Fan. Short-living supermassive magnetar model for the early X-ray flares following short GRBs. Chinese Journal of Astronomy and Astrophysics, 6(5):513-516, 2005. doi: 10.1088/1009-9271/6/5/01.

[14] Ben Margalit, Brian D. Metzger, Edo Berger, et al. Unveiling the Engines of Fast Radio Bursts, Super-Luminous Supernovae, and Gamma-Ray Bursts. Monthly Notices of the Royal Astronomical Society, 481(2):2407-2426, 2018. doi: 10.1093/mnras/sty2417.

[15] V. Savchenko, C. Ferrigno, E. Kuulkers, et al. INTEGRAL Detection of the First Prompt Gamma-Ray Signal Coincident with the Gravitational Wave Event GW170817. The Astrophysical Journal Letters, Volume 848, Issue 2, article id. L15, 8 pp. (2017)., 848, 2017. ISSN 0004-637X. doi: 10.3847/2041-8213/aa8f94.

[16] A. Goldstein, P. Veres, E. Burns, et al. An Ordinary Short Gamma-Ray Burst with Extraordinary Implications: Fermi-GBM Detection of GRB 170817A. The Astrophysical Journal, 848 (2):L14, 2017. doi: 10.3847/2041-8213/aa8f41.

[17] B.P. Abbott, R. Abbott, T.D. Abbott, et al. Gravitational Waves and Gamma-Rays from a Binary Neutron Star Merger: GW170817 and GRB 170817A. Astrophysical Journal Letters, 848(2), 2017. ISSN 20418213. doi: 10.3847/2041-8213/aa920c.

[18] C. Ferrigno, E. Kuulkers, D. Goetz, et al. INTEGRAL hard X-ray spectroscopy of AT2018cow: Preliminary detection of a cutoff at $40 \mathrm{keV}$. The Astronomer's Telegram, 11788: $1,2018$.

[19] Raffaella Margutti, B. D. Metzger, R. Chornock, et al. An embedded X-ray source shines through the aspherical AT2018cow: Revealing the inner workings of the most luminous fastevolving optical transients. The Astrophysical Journal, 872(1):18, 2018. doi: 10.3847/15384357/aafa01.

[20] V. Savchenko, C. Ferrigno, E. Kuulkers, et al. INTEGRAL observations of decaying hard X-ray emission from AT2018cow. The Astronomer's Telegram, 11843:1, 2018.

[21] S. J. Prentice, K. Maguire, S. J. Smartt, et al. The Cow: Discovery of a Luminous, Hot, and Rapidly Evolving Transient. The Astrophysical Journal, 865(1):L3, 2018. ISSN 2041-8213. doi: 10.3847/2041-8213/aadd90.

[22] Ke Fang, Brian D. Metzger, Kohta Murase, Imre Bartos, and Kumiko Kotera. Multimessenger Implications of AT2018cow: High-Energy Cosmic Ray and Neutrino Emissions from 
Magnetar-Powered Super-Luminous Transients. The Astrophysical Journal, 878(1):34, 2018. doi: 10.3847/1538-4357/ab1b72.

[23] Andrii Neronov. An online data analysis system of INTEGRAL telescope. in preparation, 2019.

[24] Volodymyr Savchenko. Astrophysical Online Data Analysis powered by provenance data model. In Proceedings of ASTERICS 2019, 2019. 